\title{
NONSTATIONARY ANALYSIS OF THE QUEUEING NETWORK WITH BYPASS OF SYSTEMS OF MULTI-TYPE AND ABSOLUTE PRIORITY MESSAGES
}

\author{
Mikhail Matalytski ${ }^{1}$, Victor Naumenko ${ }^{2}$ \\ ${ }^{I}$ Institute of Mathematics, Czestochowa University of Technology \\ Czestochowa, Poland \\ ${ }^{2}$ Faculty of Mathematics and Computer Science, Grodno State University \\ Grodno, Belarus \\ Im.matalytski@gmail.com, 2victornn86@gmail.com
}

\begin{abstract}
This paper contains an investigation of an open exponential network with bypass of systems of multi-type messages with absolute priority in transient behavior. Messages with priority received by the node with the probability depending on the state system and the type of incoming messages are for service, forcing messages that are serviced or instant changes in the following system, and can leave the network. Network settings: the intensity of the incoming flow and the intensity of service of different types of messages in single-line systems and the conditional probabilities of transitions of messages between the systems are dependent on the time. We obtained a system difference-differential equations for the state probabilities of the network. To find the state probabilities of the network in the transitional behavior applied a methodology based on the use of the apparatus of multidimensional generating functions.
\end{abstract}

Keywords: multi-type messages bypass of systems, absolute priority, the conditional probabilities of transitions, the system of difference-differential equations, probability states, generating function

\section{General information}

Results of investigations for an open network with multi-line exponential queueing systems (QS) with one-type messages bypass of systems in transient behavior were presented in [1]. In this paper, such a network was investigated, but with multi-type and absolute priority of incoming messages. If the message is received with an absolute priority in the QS, it immediately begins to serve, cutting the duration of their service request found there. The displaced thereby shall be returned to the top of the turn and the expected continuation of service (Priority Service), goes immediately into the next system or leaves the network.

In the information and telecommunication systems and networks messages with the absolute priority concerned to the operator commands Queueing Networks (QN) with bypassing of messages in the queueing systems can be used in model- 
ling, when a user sends a request to a system service, estimates how much time it will have to wait, or how many users requests in front of its in line, and depending on this evaluation remains to expect or forwards its request to another system. Such a situation may arise in the service point or points for communities.

As mentioned earlier in [1], we consider an open network of exponential QN with an arbitrary structure consisting of $n$ QS $S_{1}, S_{2}, \ldots, S_{n}$. with some probability, join the queue queueing, and with an additional probability to move immediately according to the matrix of transition probabilities to another QS or leave the network. The probability of joining the QS depends on the state of the QS and the number of QS with which the messages are sent to this QS.

The state of the network will be understood as a vector of dimension $n \times r$ :

$$
k(t)=(k, t)=\left(k_{11}, k_{12}, \ldots, k_{1 r}, k_{21}, k_{22}, \ldots, k_{2 r}, \ldots, k_{n 1}, k_{n 2}, \ldots, k_{n r}, t\right),
$$

where $k_{i c}$ - the number of messages of type $c$ at the moment $t$ in the system $S_{i}$, $k_{i}=\sum_{c=1}^{r} k_{i c}, i=\overline{1, n}$. Let $m_{i}$ - number of identical service lines in the QS $S_{i}$, $i=\overline{1, n}$. The network receives a simple flow of messages with intensity $\lambda(k, t)$, i.e. the time interval $[t, t+\Delta t)$ the network receives a message with a probability $\lambda(k, t) \Delta t+o(\Delta t)$. If at the time $t$ of service on the line $i$-th QS is located message, at the range $[t, t+\Delta t)$ of its services will end with a probability $\mu_{i c}(k, t) \Delta t+o(\Delta t)$, where $\mu_{i c}(k, t)$ - the intensity of service of messages or type $c$ at every line of system $S_{i}$ at the moment time $t, i=\overline{1, n}, c=\overline{1, r}$. In $i$-th QS the message of type $c$, independently of other of messages receives, with a probability $p_{0 \text { cic }}, i=\overline{1, n}, c=\overline{1, r}$. The message sent to this QS from the external environment at moment time $t$, having type $c$, when the network is in a state $(k, t)$, with a probability $f^{(i c)}(k, t)$, depending on the state of the $i$-th QS and type of its message, instantly becomes a service or joins the queue, if there are any, and with probability $1-f^{(i c)}(k, t)$ does not join the queue and immediately bypasses the system. Its behavior in the future, such as if it is served by this QS and has type $c$, i.e., its time of service with a probability of 1 is equal to zero. Message of type $c$ with having completed service in the $i$-th QS, independently of other of applications instantly sent to the $j$-th and the QS is the message of type $s$, with probability $p_{i c j s}$; with probability $p_{i c 0 c}$ it leaves the network $\sum_{j=1}^{n} \sum_{s=1}^{r} p_{i c j s}+p_{i c 0 c}=1, \quad i, j=\overline{1, n}$, $c, s=\overline{1, r}$. 


\section{The system of difference-differential equations for the probability of network states}

Let:

- $\varphi_{i c}(k, t)$ - the conditional probability, that the message of type $c$ is delivered to the $i$-th QS at time $t$, when the network is in a state $(k, t)$, will not be serviced by any of the QS, $i=\overline{1, n}, \overline{c=1, r}$;

- $\psi_{i c j s}(k, t)$ - the conditional probability, that the message of type $c$ is delivered to the $i$-th QS from outside at time $t$, when the network is in a state $(k, t)$, the first time, a service in $j$-th QS, received a type $s, i, j=\overline{1, n}, c, s=\overline{1, r}$;

- $\alpha_{i c}(k, t)$ - the conditional probability that the message of type $c$, served in the $i$-th queuing system at time $t$, when the network is in a state $(k, t)$, will no longer be served in any of QS, $i=\overline{1, n}, c=\overline{1, r}$;

- $\beta_{i c j s}(k, t)$ - the conditional probability that the message of type $c$, served in the $i$-th queuing system at time $t$, when the network is in state $(k, t)$ for the first time then receives services in the $j$-th QS, as a message of type $s, i, j=\overline{1, n}$, $c, s=\overline{1, r}$. It is obvious that

$$
\begin{gathered}
\varphi_{i c}(k, t)+\sum_{j=1}^{n} \sum_{s=1}^{r} \psi_{i c j s}(k, t)=1, \\
\alpha_{i c}(k, t)+\sum_{j=1}^{n} \sum_{s=1}^{r} \beta_{i c j s}(k, t)=1 \quad i, j=\overline{1, n}, c, s=\overline{1, r} .
\end{gathered}
$$

According to the formula of total probability we find that:

$$
\begin{gathered}
\varphi_{i c}(k, t)=\left(1-f^{(i c)}(k, t)\right)\left(p_{i c 0 c}+\sum_{j=1}^{n} \sum_{s=1}^{r} p_{i c j s} \varphi_{j s}(k, t)\right), \\
\alpha_{i c}(k, t)=p_{i c 0 c}+\sum_{j=1}^{n} \sum_{s=1}^{r} p_{i c j s} \varphi_{j s}\left(k-I_{i c}, t\right), \\
\psi_{i c j s}(k, t)=f^{(i c)}(k, t) \delta_{i c j s}+\left(1-f^{(i c)}(k, t)\right) \sum_{l=1}^{n} \sum_{m=1}^{r} p_{i c l m} \psi_{l m j s}(k, t), \\
\beta_{i c j s}(k, t)=\sum_{l=1}^{n} \sum_{m=1}^{r} p_{i c l m} \psi_{l m j s}\left(k-I_{i c}, t\right), i, j=\overline{1, n}, c, s=\overline{1, r},
\end{gathered}
$$


where $\delta_{i c j s}$ - Kronecker delta, which is defined as: $\delta_{i c j s}=\left\{\begin{array}{l}1, i c=j s, \\ 0, i c \neq j s\end{array}, I_{i c} \quad-\right.$ $n \times r$ - vector of zero components, except for the number of components $r(i-1)+c$, which is equal to $1, i, j=\overline{1, n}, c, s=\overline{1, r}$.

Lemma 1. Probabilities of the states considered network satisfy a system of difference-differential equations (DDE):

$$
\begin{aligned}
& \frac{d P(k, t)}{d t}=-\left[\sum_{i=1}^{n} \sum_{c=1}^{r}\left(\mu_{i c}(k, t) u\left(k_{i c}\right) \alpha_{i c}(k, t)+\lambda(k, t) p_{0 c i c} \varphi_{i c}(k, t)\right)+\right. \\
& \left.\quad+\sum_{i, j=1 c, s=1}^{n} \sum_{j s}^{r} \mu_{j,}(k, t) u\left(k_{j s}\right) \beta_{j s i c}(k, t)\right] P(k, t)+ \\
& +\sum_{i=1}^{n} \sum_{c=1}^{r} \lambda\left(k-I_{i c}, t\right) u\left(k_{i c}\right) P\left(k-I_{i c}, t\right) \sum_{j=1}^{n} \sum_{s=1}^{r} p_{0 s j s} \psi_{j s i c}\left(k-I_{i c}, t\right)+ \\
& +\sum_{i=1}^{n} \sum_{c=1}^{r} \mu_{i c}\left(k+I_{i c}, t\right) \alpha_{i c}\left(k+I_{i c}, t\right) P\left(k+I_{i c}, t\right)+ \\
& +\sum_{i=1}^{n} \sum_{c=1}^{r} \mu_{i c}\left(k+I_{i c}, t\right) \sum_{j=1}^{n} \sum_{s=1}^{r} \beta_{i c j s}\left(k+I_{i c}-I_{j s}, t\right) u\left(k_{j s}\right) P\left(k+I_{i c}-I_{j s}, t\right),
\end{aligned}
$$

where $u(x)=\left\{\begin{array}{l}1, x>0 \\ 0, x \leq 0\end{array}\right.$ - Heaviside function.

Proof. In view of the exponential service times of messages, a random process $k(t)=(k, t)$ is a Markov chain with a countable number of states. The possible transitions in the state $(k, t+\Delta t)$ for the time $\Delta t$ :

1) from the state $\left(k-I_{i c}, t\right)$ with the probability

$$
\lambda\left(k-I_{i c}, t\right) u\left(k_{i c}\right) \sum_{j=1}^{n} \sum_{s=1}^{r} p_{0 s j s} \psi_{j s i c}\left(k-I_{i c}, t\right) \Delta t+o(\Delta t), i=\overline{1, n}, c=\overline{1, r} ;
$$

2) from the state $\left(k+I_{i c}, t\right)$ with the probability

$$
\mu_{i c}\left(k+I_{i c}, t\right) \alpha_{i c}\left(k+I_{i c}, t\right) \Delta t+o(\Delta t), i=\overline{1, n}, c=\overline{1, r} ;
$$

3) from the state $\left(k+I_{i c}-I_{j s}, t\right)$ with the probability

$$
\mu_{i c}\left(k+I_{i c}, t\right) \sum_{j=1}^{n} \sum_{s=1}^{r} u\left(k_{j s}\right) \beta_{i c j s}\left(k+I_{i c}-I_{j s}, t\right) \Delta t+o(\Delta t), i=\overline{1, n}, c=\overline{1, r}
$$


4) from the state $(k, t)$ with the probability

$$
\begin{gathered}
{\left[\sum_{i=1}^{n} \sum_{c=1}^{r}\left(\mu_{i c}(k, t) u\left(k_{i c}\right) \alpha_{i c}(k, t)+\lambda(k, t) p_{0 c i c} \varphi_{i c}(k, t)\right)+\right.} \\
\left.+\sum_{i, j=1}^{n} \sum_{c, s=1}^{r} \mu_{j s}(k, t) u\left(k_{j s}\right) \beta_{j s i c}(k, t)\right] \Delta t+o(\Delta t) ;
\end{gathered}
$$

$5)$ of the remaining states with a probability $o(\Delta t)$.

Then, using the formula of total probability, we can obtain

$$
\begin{gathered}
P(k, t+\Delta t)=-P(k, t)\left[\sum_{i=1}^{n} \sum_{c=1}^{r}\left(\mu_{i c}(k, t) u\left(k_{i c}\right) \alpha_{i c}(k, t)+\lambda(k, t) p_{0 c i c} \varphi_{i c}(k, t)\right)+\right. \\
\left.+\sum_{i, j=1 c, s=1}^{n} \sum_{j s}^{r} \mu_{j s}(k, t) u\left(k_{j s}\right) \beta_{j s i c}(k, t)\right] \Delta t+ \\
+\sum_{i=1}^{n} \sum_{c=1}^{r} \lambda\left(k-I_{i c}, t\right) u\left(k_{i c}\right) P\left(k-I_{i c}, t\right) \sum_{j=1}^{n} \sum_{s=1}^{r} p_{0 s j s} \psi_{j s i c}\left(k-I_{i c}, t\right) \Delta t+ \\
+\sum_{i=1}^{n} \sum_{c=1}^{r} \mu_{i c}\left(k+I_{i c}, t\right) \alpha_{i c}\left(k+I_{i c}, t\right) P\left(k+I_{i c}, t\right) \Delta t+ \\
+\sum_{i=1}^{n} \sum_{c=1}^{r} \mu_{i c}\left(k+I_{i c}, t\right) u\left(k_{j s}\right) \sum_{j=1}^{n} \sum_{s=1}^{r} \beta_{i c j s}\left(k+I_{i c}-I_{j s}, t\right) P\left(k+I_{i c}-I_{j s}, t\right) \Delta t+o(\Delta t) .
\end{gathered}
$$

Dividing both sides of this relationship by $\Delta t$ and taking the limit $\Delta t \rightarrow 0$, we obtain a system of equations for the state probabilities of the network (6).

\section{Finding the state probabilities network}

Some restrictions are imposed on the investigated model. Let the intensity of the incoming flow $\lambda(k, t)$ and intensity of service messages of type $c$ in every line of system $S_{i}$ at time $t \mu_{i c}(k, t)$ depend only on time $t, i=\overline{1, n}, c=\overline{1, r}$. Conditional probabilities $\varphi_{i c}(k, t), \psi_{i c j s}(k, t), \alpha_{i c}(k, t), \beta_{i c j s}(k, t)$ let also not dependent on network conditions, $i, j=\overline{1, n}, c, s=\overline{1, r}$. In addition, let $m_{i}=1, i=\overline{1, n}$, and suppose, that $k_{i c}>0, \forall t>0, i=\overline{1, n}, c=\overline{1, r}$. then system of equations (6) takes the form: 


$$
\begin{gathered}
\frac{d P(k, t)}{d t}=-\left[\sum_{i=1}^{n} \sum_{c=1}^{r}\left(\mu_{i c}(t) \alpha_{i c}(t)+\lambda(t) p_{0 c i c} \varphi_{i c}(t)\right)+\right. \\
\left.+\sum_{i, j=1}^{n} \sum_{c, s=1}^{r} \mu_{j s}(t) \beta_{j s i c}(t)\right] P(k, t)+\lambda(t) \sum_{i=1}^{n} \sum_{c=1}^{r} P\left(k-I_{i c}, t\right) \sum_{j=1}^{n} \sum_{s=1}^{r} p_{0 s j s} \psi_{j s i c}(t)+ \\
+\sum_{i=1}^{n} \sum_{c=1}^{r} \mu_{i c}(t) \alpha_{i c}(t) P\left(k+I_{i c}, t\right)+ \\
+\sum_{i=1}^{n} \sum_{c=1}^{r} \mu_{i c}(t) \sum_{j=1}^{n} \sum_{s=1}^{r} \beta_{i c j s}(t) P\left(k+I_{i c}-I_{j s}, t\right)
\end{gathered}
$$

Denote by $\Psi_{n}(z, t)$, where $z=\left(z_{11}, \ldots, z_{1 r}, \ldots, z_{n 1}, \ldots, z_{n r}\right)$, generating function of the dimension $n \times r$ :

$$
\begin{aligned}
& \Psi_{n}(z, t)=\sum_{k_{11}=1}^{\infty} \ldots \sum_{k_{1 r}=1}^{\infty} \ldots \sum_{k_{n 1}=1}^{\infty} \ldots \sum_{k_{n r}=1}^{\infty} P\left(z_{11}, \ldots z_{1 r}, \ldots, z_{n 1}, \ldots, z_{n r}, t\right) \times \\
& \times z_{11}^{k_{11} \ldots} z_{1 r}^{k_{1 r}} \ldots z_{n 1}^{k_{n 1} \ldots} z_{n r}^{k_{n r}}=\sum_{k_{11}=1}^{\infty} \ldots \sum_{k_{1 r}=1}^{\infty} \ldots \sum_{k_{n 1}=1}^{\infty} \ldots \sum_{k_{n r}=1}^{\infty} P(k, t) \prod_{i=1}^{n} \prod_{c=1}^{r} z_{i c}^{k_{i c}} .
\end{aligned}
$$

Multiplying (7) by $\prod_{l=1}^{n} \prod_{m=1}^{r} z_{l m}^{k l m}$ and summing over all possible values $k_{l m}$ from 1 to $+\infty, l=\overline{1, n}$, we obtain:

$$
\begin{aligned}
& \sum_{k_{11}=1}^{\infty} \ldots \sum_{k_{1}=1}^{\infty} \ldots \sum_{k_{n 1}=1}^{\infty} \ldots \sum_{k_{n r}=1}^{\infty} \frac{d P(k, t)}{d t} \prod_{l=1}^{n} \prod_{m=1}^{r} z_{l m}^{k_{l m}}= \\
& =-\left[\sum_{i=1}^{n} \sum_{c=1}^{r}\left(\mu_{i c}(t) \alpha_{i c}(t)+\lambda(t) p_{0 c i c} \varphi_{i c}(t)\right)+\sum_{i, j=1}^{n} \sum_{c, s=1}^{r} \mu_{j s}(t) \beta_{j s i c}(t)\right] \times \\
& \times \sum_{k_{11}=1}^{\infty} \ldots \sum_{k_{1 r}=1}^{\infty} \ldots \sum_{k_{n 1}=1}^{\infty} \ldots \sum_{k_{n r}=1}^{\infty} P(k, t) \prod_{l=1}^{n} \prod_{m=1}^{r} z_{l m}^{k_{l m}}+ \\
& +\lambda(t) \sum_{i=1}^{n} \sum_{c=1}^{r} \sum_{j=1}^{n} \sum_{s=1}^{r} p_{0 s j s} \psi_{j s i c}(t) \sum_{k_{11}=1}^{\infty} \ldots \sum_{k_{1}=1}^{\infty} \ldots \sum_{k_{n 1}=1}^{\infty} \ldots \sum_{k_{n r}=1}^{\infty} P\left(k-I_{i c}, t\right) \prod_{l=1}^{n} \prod_{m=1}^{r} z_{l m}^{k_{l} m}+ \\
& +\sum_{i=1}^{n} \sum_{c=1}^{r} \mu_{i c}(t) \alpha_{i c}(t) \sum_{k_{11}=1}^{\infty} \ldots \sum_{k_{1 r}=1}^{\infty} \ldots \sum_{k_{n 1}=1}^{\infty} \ldots \sum_{k_{n r}=1}^{\infty} P\left(k+I_{i c}, t\right) \prod_{l=1}^{n} \prod_{m=1}^{r} z_{l m}^{k_{l m}}+ \\
& +\sum_{i=1}^{n} \sum_{c=1}^{r} \mu_{i c}(t) \sum_{\substack{j=1 \\
j \neq i}}^{n} \sum_{s=1}^{r} \beta_{i c j s}(t) \times \\
& \times \sum_{k_{11}=1}^{\infty} \ldots \sum_{k_{1 r}=1}^{\infty} \ldots \sum_{k_{n 1}=1}^{\infty} \ldots \sum_{k_{n r}=1}^{\infty} P\left(k+I_{i c}-I_{j s}, t\right) \prod_{l=1}^{n} \prod_{m=1}^{r} z_{l m}^{k_{l m}}
\end{aligned}
$$


Consider some of the amounts included to the right side of relations (8). Let

$$
\sum_{1}(z, t)=\lambda(t) \sum_{i=1}^{n} \sum_{c=1}^{r} \sum_{j=1}^{n} \sum_{s=1}^{r} p_{0 s j s} \psi_{j s i c}(t) \sum_{k_{11}=1}^{\infty} \ldots \sum_{k_{1 r}=1}^{\infty} \ldots \sum_{k_{n 1}=1}^{\infty} \ldots \sum_{k_{n r}=1}^{\infty} P\left(k-I_{i c}, t\right) \prod_{l=1}^{n} \prod_{m=1}^{r} z_{l m}^{k_{l m}} .
$$

Then

$$
\begin{gathered}
\sum_{1}(z, t)=\lambda(t) \sum_{i=1}^{n} \sum_{c=1}^{r} \sum_{j=1}^{n} \sum_{s=1}^{r} p_{0 s j s} \psi_{j s i c}(t) z_{i c} \sum_{k_{11}=1}^{\infty} \ldots \sum_{k_{1 r}=1}^{\infty} \ldots \sum_{k_{n 1}=1}^{\infty} \ldots \sum_{k_{n r}=1}^{\infty} P\left(k-I_{i c}, t\right) \prod_{l=1}^{n} \prod_{m=1}^{r} z_{l m}^{k_{l m}} z_{i c}^{k_{i c}-1}= \\
=\lambda(t) \sum_{i=1}^{n} \sum_{c=1}^{r} \sum_{j=1}^{n} \sum_{s=1}^{r} p_{0 s j s} \psi_{j s i c}(t) z_{i c} \sum_{k_{11}=1}^{\infty} \ldots \sum_{k_{1 r}=1}^{\infty} \ldots \sum_{k_{n 1}=1}^{\infty} \ldots \sum_{k_{n r}=1}^{\infty} P(k, t) \prod_{l=1}^{n} \prod_{m=1}^{r} z_{l m}^{k_{l m}}= \\
=\lambda(t) \sum_{i=1}^{n} \sum_{c=1}^{r} \sum_{j=1}^{n} \sum_{s=1}^{r} p_{0 s j s} \psi_{j s i c}(t) z_{i c} \Psi_{n}(z, t) .
\end{gathered}
$$

For the sum

$$
\sum_{2}(z, t)=\sum_{i=1}^{n} \sum_{c=1}^{r} \mu_{i c}(t) \alpha_{i c}(t) \sum_{k_{11}=1}^{\infty} \ldots \sum_{k_{1}=1}^{\infty} \ldots \sum_{k_{n 1}=1}^{\infty} \ldots \sum_{k_{n r}=1}^{\infty} P\left(k+I_{i c}, t\right) \prod_{l=1}^{n} \prod_{m=1}^{r} z_{l m}^{k_{l m}}
$$

having:

$$
\begin{gathered}
\sum_{2}(z, t)=\sum_{i=1}^{n} \sum_{c=1}^{r} \mu_{i c}(t) \alpha_{i c}(t) \frac{1}{z_{i c}} \sum_{k_{11}=1}^{\infty} \ldots \sum_{k_{1}=1}^{\infty} \ldots \sum_{k_{n 1}=1}^{\infty} \ldots \sum_{k_{n r}=1}^{\infty} P\left(k+I_{i c}, t\right) \prod_{l=1}^{n} \prod_{m=1}^{r} z_{l m}^{k_{l m}} z_{i c}^{k_{i c}+1}= \\
=\sum_{i=1}^{n} \sum_{c=1}^{r} \mu_{i c}(t) \alpha_{i c}(t) \frac{1}{z_{i c}} \sum_{k_{11}=1}^{\infty} \ldots \sum_{k_{1}=1}^{\infty} \ldots \sum_{k_{n 1}=1}^{\infty} \ldots \sum_{k_{n r}=1}^{\infty} P(k, t) \prod_{l=1}^{n} \prod_{m=1}^{r} z_{l m}^{k_{l m}} z_{i c}^{k_{i c}+1}= \\
=\sum_{i=1}^{n} \sum_{c=1}^{r} \mu_{i c}(t) \alpha_{i c}(t) \frac{1}{z_{i c}}- \\
-\sum_{i=1}^{n} \sum_{c=1}^{r} \mu_{i c}(t) \alpha_{i c}(t) \frac{1}{z_{i c}} \sum_{\substack{k_{j s}=1 \\
j=1, n, j \neq i, s=1, r, s \neq c}}^{\infty} P\left(k_{11}, \ldots, k_{1 r}, k_{i-1, c}, 0, k_{i+1, c}, . ., k_{n r}, t\right) \prod_{\substack{l=1 \\
l \neq i}}^{n} \prod_{m=1}^{r} z_{l}^{k_{l m}}= \\
=\sum_{i=1}^{n} \sum_{c=1}^{r} \mu_{i c}(t) \alpha_{i c}(t) \frac{1}{z_{i c}} \Psi_{n}(z, t),
\end{gathered}
$$

because by assumption $k_{i c}>0$, i.e. $P\left(k_{11}, \ldots, k_{1 r}, k_{i-1, c}, 0, k_{i+1, c}, . ., k_{n r}, t\right)=0$, $i=\overline{1, n}, c=\overline{1, r}$.

And finally, for the last sum

$$
\sum_{3}(z, t)=\sum_{i=1}^{n} \sum_{c=1}^{r} \mu_{i c}(t) \sum_{\substack{j=1 \\ j \neq i}}^{n} \sum_{\substack{s=1 \\ S \neq c}}^{r} \beta_{i c j s}(t) \sum_{k_{11}=1}^{\infty} \ldots \sum_{k_{1}=1}^{\infty} \ldots \sum_{k_{n 1}=1}^{\infty} \ldots \sum_{k_{n r}=1}^{\infty} P\left(k+I_{i c}-I_{j s}, t\right) \prod_{l=1}^{n} \prod_{m=1}^{r} z_{l m}^{k_{l m}}
$$


we have:

$$
\begin{gathered}
\sum_{3}(z, t)=\sum_{i=1}^{n} \sum_{c=1}^{r} \mu_{i c}(t) \sum_{j=1}^{n} \sum_{s=1}^{r} \beta_{i c j s}(t) \frac{z_{j s}}{z_{i c}} \times \\
\times \sum_{\substack{k_{j s}=1 \\
j=1, n, j \neq i, j}}^{\infty} \sum_{\substack{j s \\
s=1, r, s \neq c}}^{\infty} P\left(k_{11}, \ldots, k_{1 r}, k_{i-1, c}, k_{i c}+1, k_{i+1, c}, \ldots, k_{j-1, s}, k_{j s}-1, k_{j+1, s}, . ., k_{n r}, t\right) \times \\
\times \prod_{\substack{l=1 \\
l \neq i, j}}^{n} \prod_{m=1}^{r} z_{l}^{k_{l m}} z_{i c}^{k_{l c}+1} z_{j s}^{k_{j s}-1}= \\
=\sum_{i=1}^{n} \sum_{c=1}^{r} \mu_{i c}(t) \sum_{j=1}^{n} \sum_{s=1}^{r} \beta_{i c j s}(t) \frac{z_{j s}}{z_{i c}} \sum_{k_{11}=1}^{\infty} \ldots \sum_{k_{1}=1}^{\infty} \ldots \sum_{k_{n 1}=1}^{\infty} \ldots \sum_{k_{n r}=1}^{\infty} P(k, t) \prod_{l=1}^{n} \prod_{m=1}^{r} z_{l m}^{k_{l m}}= \\
=\sum_{i=1}^{n} \sum_{c=1}^{r} \mu_{i c}(t) \sum_{j=1}^{n} \sum_{s=1}^{r} \beta_{i c j s}(t) \frac{z_{j s}}{z_{i c}} \Psi_{n}(z, t)- \\
-\sum_{i=1}^{n} \sum_{c=1}^{r} \mu_{i c}(t) \sum_{j=1}^{n} \sum_{s=1}^{r} \beta_{i c j s}(t) \frac{z_{j s}}{z_{i c}} \sum_{\substack{k_{j s}=1 \\
j=1, n, j \neq i,}}^{\infty} P\left(k_{11}, \ldots, k_{1 r}, k_{i-1, c}, 0, k_{i+1, c}, \ldots, k_{n r}, t\right) \prod_{\substack{l=1 \\
l \neq i}}^{n} \prod_{m=1}^{r} z_{l}^{k_{l m}}= \\
=\sum_{i=1}^{n} \sum_{c=1}^{r} \mu_{i c}(t) \sum_{j=1}^{n} \sum_{s=1}^{r} \beta_{i c j s}(t) \frac{z_{j s}}{z_{i c}} \Psi_{n}(z, t),
\end{gathered}
$$

for the same reasons as for the sum of $\sum_{2}(z, t)$.

Thus, for the generating function we obtain a homogeneous linear differential equation

$$
\begin{gathered}
\frac{d \Psi_{n}(z, t)}{d t}=\left\{-\left[\sum_{i=1}^{n} \sum_{c=1}^{r}\left(\mu_{i c}(t) \alpha_{i c}(t)+\lambda(t) p_{0 c i c} \varphi_{i c}(t)\right)+\sum_{i, j=1}^{n} \sum_{c, s=1}^{r} \mu_{j s}(t) \beta_{j s i c}(t)\right]+\right. \\
+\lambda(t) \sum_{i=1}^{n} \sum_{c=1}^{r} \sum_{j=1}^{n} \sum_{s=1}^{r} p_{0 s j s} \psi \psi_{j s i c}(t) z_{i c}+\sum_{i=1}^{n} \sum_{c=1}^{r} \mu_{i c}(t) \alpha_{i c}(t) \frac{1}{z_{i c}}+ \\
\left.+\sum_{i=1}^{n} \sum_{c=1}^{r} \mu_{i c}(t) \sum_{j=1}^{n} \sum_{s=1}^{r} \beta_{i c j s}(t) \frac{z_{j s}}{z_{i c}}\right\} \Psi_{n}(z, t)
\end{gathered}
$$

Its solution has the form

$$
\begin{aligned}
& \Psi_{n}(z, t)=C_{n}(z) \exp \left\{\int\right.-\left[\sum_{i=1}^{n} \sum_{c=1}^{r}\left(\mu_{i c}(t) \alpha_{i c}(t)+\lambda(t) p_{0 c i c} \varphi_{i c}(t)\right)+\sum_{i, j=1 c, s=1}^{n} \sum_{j s}^{r} \mu_{j s}(t) \beta_{j s i c}(t)\right]+ \\
&+\lambda(t) \sum_{i=1}^{n} \sum_{c=1}^{r} \sum_{j=1}^{n} \sum_{s=1}^{r} p_{0 s j s} \psi_{j s i c}(t) z_{i c}+\sum_{i=1}^{n} \sum_{c=1}^{r} \mu_{i c}(t) \alpha_{i c}(t) \frac{1}{z_{i c}}+ \\
&\left.\left.+\sum_{i=1}^{n} \sum_{c=1}^{r} \mu_{i c}(t) \sum_{j=1}^{n} \sum_{s=1}^{r} \beta_{i c j s}(t) \frac{z_{j s}}{z_{i c}}\right] d t\right\}
\end{aligned}
$$


We assume that at the initial time the network is in a state

$$
\begin{gathered}
\left(x_{11}, \ldots, x_{1 r}, x_{21}, \ldots, x_{2 r}, \ldots, x_{n 1}, \ldots, x_{n r}, 0\right), x_{i c}>0, i=\overline{1, n}, c=\overline{1, r}, \\
P\left(x_{11}, \ldots, x_{1 r}, x_{21}, \ldots, x_{2 r}, \ldots, x_{n 1}, \ldots, x_{n r}, 0\right)=1, \\
P\left(k_{11}, k_{12}, \ldots, k_{1 r}, k_{21}, k_{22}, \ldots, k_{2 r}, \ldots, k_{n 1}, k_{n 2}, \ldots, k_{n r}, 0\right)=0,
\end{gathered}
$$

$\forall x_{i c} \neq k_{i c}, i=\overline{1, n}, c=\overline{1, r}$. Then the initial condition for the last equation (10) will be

$$
\Psi_{n}(z, 0)=P\left(x_{11}, \ldots, x_{1 r}, x_{21}, \ldots, x_{2 r}, \ldots, x_{n 1}, \ldots, x_{n r}, 0\right) \prod_{l=1}^{n} \prod_{m=1}^{r} z_{l m}^{k_{l m}}=\prod_{l=1}^{n} \prod_{m=1}^{r} z_{l m}^{k_{l m}}
$$

Using this initial condition, we obtain

$$
\begin{aligned}
C_{n}(z)=\exp \left\{\int \left[\left[\sum_{i=1}^{n} \sum_{c=1}^{r}\left(\mu_{i c}(t) \alpha_{i c}(t)+\lambda(t) p_{0 c i c} \varphi_{i c}(t)\right)+\sum_{i, j=1}^{n} \sum_{c, s=1}^{r} \mu_{j s}(t) \beta_{j s i c}(t)\right]+\right.\right. \\
+\lambda(t) \sum_{i=1}^{n} \sum_{c=1}^{r} \sum_{j=1}^{n} \sum_{s=1}^{r} p_{0 s j s} \psi_{j s i c}(t) z_{i c}+\sum_{i=1}^{n} \sum_{c=1}^{r} \mu_{i c}(t) \alpha_{i c}(t) \frac{1}{z_{i c}}+ \\
\left.\left.+\sum_{i=1}^{n} \sum_{c=1}^{r} \mu_{i c}(t) \sum_{j=1}^{n} \sum_{s=1}^{r} \beta_{i c j s}(t) \frac{z_{j s}}{z_{i c}}\right]\left.d t\right|_{t=0}\right\} \prod_{l=1}^{n} \prod_{m=1}^{r} z_{l m}^{k_{l m}}
\end{aligned}
$$

Thus, we have the assertion which can be summarized as follows:

Lemma 2. If at the initial moment of time $Q N$ is in a state $\left(x_{11}, \ldots, x_{1 r}, x_{21}, \ldots, x_{2 r}, \ldots, x_{n 1}, \ldots, x_{n r}, 0\right), x_{i c}>0, i=\overline{1, n}, c=\overline{1, r}$, then the expression for $\Psi_{n}(z, t)$ can be rewritten as (10), where the function $C_{n}(z)$ has the form (11).

We introduce the following notation:

$$
\begin{gathered}
\Lambda(t)=\int \lambda(t) d t, M_{i c}(t)=\int \mu_{i c}(t) d t, \Phi_{i c}(t)=\int \lambda(t) \varphi_{i c}(t) d t, \\
\mathrm{~A}_{i c}(t)=\int \mu_{i c}(t) \alpha_{i c}(t) d t, \mathrm{~B}_{i c j s}(t)=\int \mu_{i c}(t) \beta_{i c j s}(t) d t, \\
\mathrm{Y}_{i c j s}(t)=\int \lambda(t) \psi_{i c j s}(t) d t .
\end{gathered}
$$

Then, taking into account input notation, we obtain that (12) becomes:

$$
\begin{aligned}
& \Psi_{n}(z, t)= \exp \left\{-\left[\sum_{i=1}^{n} \sum_{c=1}^{r}\left(\left(\mathrm{~A}_{i c}(t)-\mathrm{A}_{i c}(0)\right)+p_{0 c i c}\left(\Phi_{i c}(t)-\Phi_{i c}(0)\right)\right)+\sum_{i, j=1 c, s=1}^{n} \sum_{j s i c}^{r}(t)-\mathrm{B}_{j s i c}(0)\right)\right]+ \\
&+ \sum_{i, j=1}^{n} \sum_{c, s=1}^{r} p_{0 s j s}\left(\mathrm{Y}_{j s i c}(t)-\mathrm{Y}_{j s i c}(0)\right) z_{i c}+\sum_{i=1}^{n} \sum_{c=1}^{r}\left(A_{i c}(t)-A_{i c}(0)\right) \frac{1}{z_{i c}}+ \\
&\left.+\sum_{i, j=1}^{n} \sum_{c, s=1}^{r}\left(B_{i c j s}(t)-B_{i c j s}(0)\right) \frac{z_{j s}}{z_{i c}}\right\} \prod_{l=1}^{n} \prod_{m=1}^{r} z_{l m}^{x_{l m}} .
\end{aligned}
$$


The last expression can be rewritten as:

$$
\begin{aligned}
& \Psi_{n}(z, t)=a_{0}(t) \exp \left\{\sum_{i=1}^{n} \sum_{c=1}^{r} \sum_{j=1}^{n} \sum_{s=1}^{r} p_{0 s j s}\left(Y_{j s i c}(t)-Y_{j s i c}(0)\right) z_{i c}\right\} \exp \left\{\sum_{i=1}^{n} \sum_{c=1}^{r}\left(A_{i c}(t)-A_{i c}(0)\right) \frac{1}{z_{i c}}\right\} \times \\
& \times \exp \left\{\sum_{i=1}^{n} \sum_{c=1}^{r} \sum_{\substack{j=1 \\
j \neq i}}^{n} \sum_{s=1}^{r}\left(\mathrm{~B}_{i c j s}(t)-\mathrm{B}_{i c j s}(0)\right) \frac{z_{j s}}{z_{i c}}\right\} \prod_{l=1}^{n} \prod_{m=1}^{r} z_{l m}^{x_{l m}}, \\
& a_{0}(t)=\exp \left\{-\left[\sum_{i=1}^{n} \sum_{c=1}^{r}\left(\left(\mathrm{~A}_{i c}(t)-\mathrm{A}_{i c}(0)\right)+p_{0 c i c}\left(\Phi_{i c}(t)-\Phi_{i c}(0)\right)\right)+\right.\right. \\
& \left.\left.+\sum_{i, j=1}^{n} \sum_{c, s=1}^{r}\left(\mathrm{~B}_{j s i c}(t)-\mathrm{B}_{j s i c}(0)\right)\right]\right\}
\end{aligned}
$$

Transform (13) to a form suitable for finding the state probabilities of the network are expand its member exhibitors in a Maclaurin series. Then the following statement

Theorem. The expression for the generating function has the form

$$
\begin{gathered}
\Psi_{n}(z, t)=a_{0}(t) \sum_{l_{1}=0}^{\infty} \ldots \sum_{l_{n}=0}^{\infty} \sum_{q_{1}=0}^{\infty} \ldots \sum_{q_{n}=0}^{\infty} \sum_{w_{1}=0}^{\infty} \ldots \sum_{w_{n}=0}^{\infty} \prod_{i=1}^{n} \prod_{c=1}^{r} \prod_{j=1}^{n} \prod_{s=1}^{r}\left(\frac{p_{0 s j s}^{l_{i}}}{l_{i} ! q_{i} ! w_{i} !} \times\right. \\
\left.\times\left[Y_{j s i c}(t)-Y_{j s i c}(0)\right]^{l_{i}}\left[A_{i c}(t)-A_{i c}(0)\right]^{q_{i}}\left[\mathrm{~B}_{i c j s}(t)-\mathrm{B}_{i c j s}(0)\right]^{w_{i}} z_{i c}^{x_{i}+l_{i}-q_{i}+W}\right), \quad \text { (15) }
\end{gathered}
$$

where $W=\sum_{i=1}^{n} w_{i}$.

Proof. From the relations (13) and (14) and Lemma 2 follows that

$$
\Psi_{n}(z, t)=a_{0}(t) a_{1}(z, t) a_{2}(z, t) a_{3}(z, t) \prod_{l=1}^{n} \prod_{m=1}^{r} z_{l m}^{x_{l m}},
$$

where

$$
\begin{gathered}
a_{1}(z, t)=\exp \left\{\sum_{i=1}^{n} \sum_{c=1}^{r} \sum_{j=1}^{n} \sum_{s=1}^{r} p_{0 s j s}\left(Y_{j s i c}(t)-Y_{j s i c}(0)\right) z_{i c}\right\}= \\
=\prod_{i=1}^{n} \prod_{c=1}^{r} \prod_{\substack{j=1 \\
j \neq i}}^{n} \prod_{\substack{s=1 \\
s \neq c}}^{r} \exp \left\{p_{0 s j s}\left(Y_{j s i c}(t)-Y_{j s i c}(0)\right) z_{i c}\right\}=
\end{gathered}
$$




$$
\begin{aligned}
& =\prod_{i=1}^{n} \prod_{c=1}^{r} \prod_{\substack{j=1 \\
j \neq i}}^{n} \prod_{\substack{s=1 \\
s \neq c}}^{r} \sum_{l_{i}}^{\infty} \frac{\left[p_{0 s j s}\left(Y_{j s i c}(t)-Y_{j s i c}(0)\right) z_{i c}\right]^{l_{i}}}{l_{i} !}=
\end{aligned}
$$

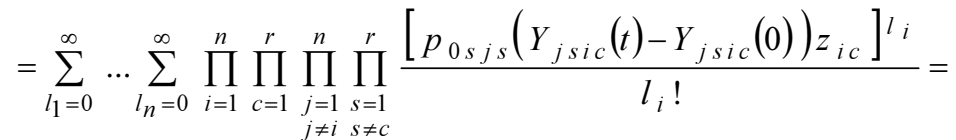

$$
\begin{aligned}
& =\sum_{l_{1}=0}^{\infty} \ldots \sum_{l_{n}=0}^{\infty} \frac{p_{0111}^{l_{1}} \cdots p_{0 r n r}^{l_{n}} z_{11}^{l_{1}} \cdots z_{1 r}^{l_{1}} \cdots z_{n r}^{l_{n}}}{l_{1} ! l_{2} ! \cdots l_{n} !} \prod_{i=1}^{n} \prod_{c=1}^{r} \prod_{\substack{j=1 \\
j \neq i}}^{n} \prod_{\substack{s \neq 1 \\
\text { s }}}^{r}\left[Y_{j s i c}(t)-Y_{j s i c}(0)\right]^{l_{i}}, \\
& a_{2}(z, t)=\exp \left\{\sum_{i=1}^{n} \sum_{c=1}^{r} \frac{1}{z_{i c}}\left(\mathrm{~A}_{i c}(t)-\mathrm{A}_{i c}(0)\right)\right\}=\prod_{i=1}^{n} \prod_{c=1}^{r} \exp \left\{\frac{1}{z_{i c}}\left(\mathrm{~A}_{i c}(t)-\mathrm{A}_{i c}(0)\right)\right\}= \\
& =\prod_{i=1}^{n} \prod_{c=1}^{r} \sum_{q_{i}=0}^{\infty} \frac{\left[\left(\mathrm{A}_{i c}(t)-\mathrm{A}_{i c}(0)\right) z_{i c}^{-1}\right]^{q_{i}}}{q_{i} !}=\sum_{q_{1}=0}^{\infty} \ldots \sum_{q_{n}=0}^{\infty} \prod_{i=1}^{n} \prod_{c=1}^{r} \frac{\left[\left(\mathrm{A}_{i c}(t)-\mathrm{A}_{i c}(0)\right) z_{i c}^{-1}\right]^{q_{i}}}{q_{i} !}= \\
& =\sum_{q 1=0}^{\infty} \ldots \sum_{q_{n}=0}^{\infty} \frac{\prod_{i=1}^{n} \prod_{c=1}^{r}\left[\mathrm{~A}_{i c}(t)-\mathrm{A}_{i c}(0)\right]^{q i}}{q_{1} ! \cdots \cdot q_{n} !} z_{11}^{-q_{1}} \cdots z_{1 r}^{-q_{1}} \cdots z_{n 1}^{-q_{n}} \cdots z_{n r}^{-q_{n}}, \\
& a_{3}(z, t)=\exp \left\{\sum_{i=1}^{n} \sum_{c=1}^{r} \sum_{\substack{j=1 s \\
j \neq i s \neq c}}^{n} \sum_{j=1}^{r}\left(\mathrm{~B}_{i c j s}(t)-\mathrm{B}_{i c j s}(0)\right) \frac{z_{j s}}{z_{i c}}\right\}=\prod_{i=1}^{n} \prod_{c=1}^{r} \prod_{\substack{j=1 s=1 \\
j \neq i s \neq c}}^{n} \prod_{\substack{r \\
j}}^{r} \exp \left\{\frac{z_{j s}}{z_{i c}}\left(\mathrm{~B}_{i c j s}(t)-\mathrm{B}_{i c j s}(0)\right)\right\}= \\
& =\prod_{i=1}^{n} \prod_{c=1}^{r} \prod_{\substack{j=1 \\
j \neq i}}^{n} \prod_{\substack{s=1 \\
s \neq c}}^{r} \sum_{w_{i}}^{\infty} \frac{\left[\left(\mathrm{B}_{i c j s}(t)-\mathrm{B}_{i c j s}(0)\right) z_{j s} z_{i c}{ }^{-1}\right]^{w_{i}}}{w_{i} !}=
\end{aligned}
$$

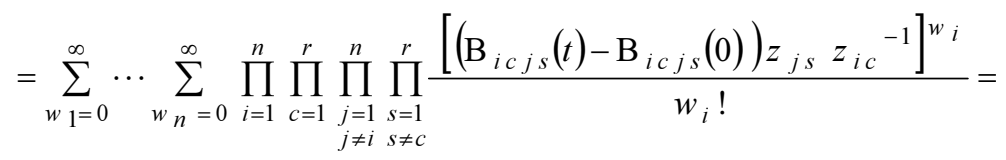

$$
\begin{aligned}
& =\sum_{w_{1}=0}^{\infty} \cdots \sum_{w_{n}=0}^{\infty} \frac{\prod_{i=1}^{n} \prod_{c=1}^{r}\left(\prod_{j=1}^{n} \prod_{s=1}^{r}\left(\mathrm{~B}_{i c j s}(t)-\mathrm{B}_{i c j s}(0)\right)\right)^{w_{i}}}{w_{1} ! \cdots w_{n} !} \times \\
& \times z_{11}^{w_{1}+w_{2}+\ldots+w_{n}} \cdots z_{n_{r}}^{w_{1}+w_{2}+\ldots+w_{n}} z_{11}^{-w_{1}} \cdots z_{n r}^{-w_{n}}= \\
& =\sum_{w_{1}=0}^{\infty} \cdots \sum_{w_{n}=0}^{\infty} \frac{\prod_{i=1}^{n} \prod_{c=1}^{r}\left(\prod_{j=1}^{n} \prod_{s=1}^{r}\left(\mathrm{~B}_{i c j s}(t)-\mathrm{B}_{i c j s}(0)\right)\right)^{w_{i}}}{w_{1} ! \cdots w_{n} !} z_{11}^{W-w_{1}} \cdots z_{1 r}^{W-w_{1}} \cdots z_{n 1}^{W-w_{n}} \cdots z_{n r}^{W-w_{n}} .
\end{aligned}
$$

Multiplying $a_{0}(t), a_{1}(z, t), a_{2}(z, t), a_{3}(z, t)$ and $\prod_{l=1}^{n} \prod_{m=1}^{r} z_{l m}^{x_{l m}}$ we obtain the expression for the generating function of the form (15). 


\section{Example}

Let the intensity of the input and services of messages have the form $\lambda(t)=\lambda t$, $\mu_{i c}(t)=\mu_{i c}(\cos t+1), \quad i=\overline{1, n}, \quad c=\overline{1, r}$. In this case $\Lambda(t)=\frac{\lambda}{2} t^{2}, \Lambda(0)=0$, $M_{i c}(t)=\mu_{i c}(\sin t+t), M_{i c}(0)=0, i=\overline{1, n}, c=\overline{1, r}$. Assume that the probability of the messages to join the queue at the time $t$ has the form $f^{(i c)}(t)=1-e^{-i c t}$. $\Phi_{i c}(0)=0, \mathrm{Y}_{i c j s}(0)=0, \mathrm{~A}_{i c}(0)=0, \mathrm{~B}_{i c j s}(0)=\mathrm{B}_{i c j s}(0)=0, i, j=\overline{1, n}, c, s=\overline{1, r}$. Conditional probabilities $\varphi_{i c}(t), \psi_{i c j s}(t), \alpha_{i c}(t)$ and $\beta_{i c j s}(t)$ are found from (2) - (5). Solving a system of linear equations (2-5) in the package Mathematica, you can get their analytical solutions.

Expression (15) takes the form:

$$
\begin{gathered}
\Psi_{n}(z, t)=a_{0}(t) \sum_{l_{1}=0}^{\infty} \ldots \sum_{l_{n}=0}^{\infty} \sum_{q_{1}=0}^{\infty} \ldots \sum_{q_{n}=0}^{\infty} \sum_{w_{1}=0}^{\infty} \ldots \sum_{w_{n}=0}^{\infty} \prod_{i=1}^{n} \prod_{c=1}^{r} \prod_{j=1}^{n} \prod_{s=1}^{r}\left(\frac{\lambda}{2}\right)^{l_{i}}\left(\frac{\mu_{i c}{ }^{q_{i}+w_{i}} p_{0 s j s}^{l_{i}}}{l_{i} ! q_{i} ! w_{i} !} \times\right. \\
\times\left[\psi_{j s i c}(t) t^{2}-\int \psi_{j s i c}^{\prime}(t) t^{2} d t\right]^{l_{i}}\left[\alpha_{i c}(t)(\sin t+t)-\int \alpha_{i c}^{\prime}(t)(\sin t+t) d t\right]^{q_{i}} \times \\
\left.\times\left[\beta_{i c j s}(t)(\sin t+t)-\int \beta_{i c j s}^{\prime}(t)(\sin t+t) d t\right] w_{i}^{w_{i}} z_{i c}^{x_{i}+l_{i}-q_{i}+W}\right)
\end{gathered}
$$

Suppose that we need to find, for example, the probability of state $P(1,1, \ldots, 1, t)$, i.e. $k_{i c}=1, i=\overline{1, n}, c=\overline{1, r}$. It is the coefficient of $z_{11}, \ldots, z_{1 r}, \ldots, z_{n 1}, \ldots, z_{n r}$ in the expansion of $\Psi_{n}(z, t)$ in multiple series (16), so the degree for $z_{i c}$ must satisfy the relation $x_{i}+l_{i}-q_{i}+W=1, i=\overline{1, n}$. Then

$$
\begin{gathered}
P(1,1, \ldots, 1, t)=a_{0}(t) \sum_{l_{1}=0}^{\infty} \ldots \sum_{l_{n}=0}^{\infty} \sum_{w_{1}=0}^{\infty} \ldots \sum_{w_{n}=0}^{\infty} \prod_{i=1}^{n} \prod_{c=1}^{r} \prod_{j=1}^{n} \prod_{s=1}^{r}\left(\frac{\lambda}{2}\right)^{l_{i}}\left(\frac{\mu_{i c}^{x_{i}+l_{i}+\sum_{j=1} w_{j}-1} p_{0 s j s}^{l_{i}}}{\left(x_{i}+l_{i}+\sum_{\substack{j=1 \\
j \neq i}} w_{j}-1\right) ! w_{i} !} \times\right. \\
\times\left[\psi_{j s i c}(t) t^{2}-\int \psi_{j s i c}^{\prime}(t) t^{2} d t\right]^{l_{i}}\left[\alpha_{i c}(t)(\sin t+t)-\int \alpha_{i c}^{\prime}(t)(\sin t+t) d t\right]^{x_{i}+l_{i}+\sum_{\substack{j=1 \\
j \neq i}} w_{j}-1} \times \\
\left.\times\left[\beta_{i c j s}(t)(\sin t+t)-\int \beta_{i c j s}^{\prime}(t)(\sin t+t) d t\right]^{w_{i}} z_{i c}^{x_{i}+l_{i}+W-1}\right) .
\end{gathered}
$$

Let $n=9$, number of types of messages $r=6$. Suppose also that the intensity $\lambda(t)=\lambda t, \lambda=10 ; \mu_{i c}(t)=\mu_{i c}(\cos t+1), \mu_{11}=10, \mu_{22}=3, \mu_{33}=2, \mu_{44}=1$, 
$\mu_{51}=0.5, \mu_{62}=1, \mu_{75}=10, \mu_{76}=2, \mu_{86}=3, \mu_{91}=0.3, \mu_{92}=6, \mu_{93}=20$, $\mu_{94}=13$, other intensity messsages service equal to zero. The transition probabilities between QN of messages are: $p_{0191}=1, p_{0292}=1, p_{0393}=1, p_{0494}=1$, $p_{1151}=0.3, \quad p_{1191}=0.2, \quad p_{1192}=0.1, \quad p_{1193}=0.4, \quad p_{1194}=0, \quad p_{2262}=0.1$, $p_{2291}=0.2, p_{2292}=0.3, p_{2293}=0.4, p_{2294}=0, p_{3375}=0.1, \quad p_{3386}=0.1$, $p_{3391}=0.3, p_{3392}=0.4, p_{3393}=0, \quad p_{3394}=0.1, \quad p_{4491}=0.7, \quad p_{4492}=0.1$, $p_{4493}=0, p_{4494}=0.2, p_{5111}=1, p_{6222}=1, p_{7533}=1, p_{7633}=1, p_{8676}=1$, $p_{9111}=0.5, p_{9222}=0.5, p_{9333}=0.5, p_{9444}=0.5, p_{9303}=0.5, p_{9404}=0.5$, other transition probabilities are 0 . Consider the time period $t \in[0, T], T=10$.

The expression for the time-dependent probability of the state in network systems obtained by computer using the mathematical calculations package Mathematica. Figure 1 shows a graph of the probabilities depending on the time $t$.

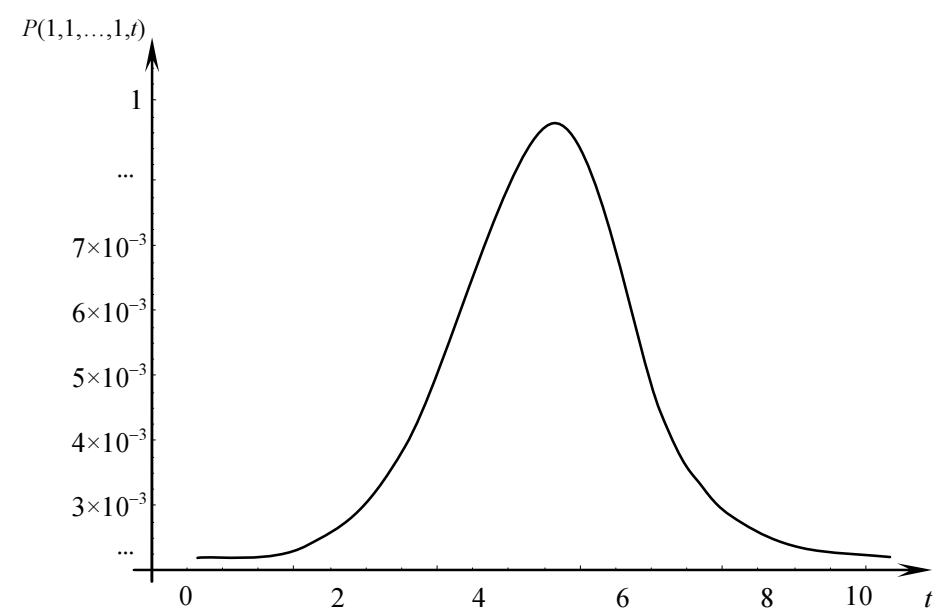

Fig. 1 . The chart of the probability of the state $P(1,1, \ldots, 1, t)$

\section{References}

[1] Matalytski M., Naumenko, V. Analysis of the queueing network with messages bypass of systems in transient behavior, Scientific Research of the Institute of Mathematics and Computer Science, Czestochowa University of Technology 2012, 11, 2, 71-83. 